\title{
An Enhanced Three-Band Index for Estimating Chlorophyll-a in Turbid Case-II Waters: Case Studies of Lake Kasumigaura, Japan, and Lake Dianchi, China
}

\author{
Wei Yang, Bunkei Matsushita, Jin Chen, Takehiko Fukushima, and Ronghua Ma
}

\begin{abstract}
A three-band index was previously proposed and successfully utilized to estimate the chlorophyll-a concentration (Chl-a) in case-II waters. However, this index shows uncertainties in highly turbid situations. In this study, an enhanced three-band index is proposed to solve this problem. Since the new index employs bands that are identical to those of the original threeband index, it can be applied to Medium Resolution Imaging Spectrometer (MERIS) data. The performance of the index was evaluated using the data collected from two turbid Asian lakes: Lake Kasumigaura, Japan, and Lake Dianchi, China. The results showed that the Chl-a predicted by the enhanced threeband index was strongly correlated with the measured Chl-a $\left(R^{2}>0.83\right)$, and the root-mean-square error (rmse) and the normalized root-mean-square error (NRMS) were both reduced for the two lakes (for Lake Kasumigaura, rmse from 13.97 to $8.68 \mathrm{mg} \cdot \mathrm{m}^{-3}$ and NRMS from $19.01 \%$ to $12.30 \%$; for Lake Dianchi, rmse from 41.29 to $15.28 \mathrm{mg} \cdot \mathrm{m}^{-3}$ and NRMS from $35.83 \%$ to $21.34 \%$ ). These findings imply that, if accurately atmospheric-corrected MERIS data are available, the enhanced three-band index could be used for mapping Chl-a even in highly turbid case-II waters.
\end{abstract}

Index Terms-Chlorophyll-a, four-band index, highly turbid case-II waters, Medium Resolution Imaging Spectrometer (MERIS) data, three-band index.

\section{INTRODUCTION}

C HLOROPHYLL-A is an important optically active substance (OAS) existing in water bodies; its concentration is strongly related to the total primary production of water bodies and also acts as an index of eutrophication with respect to water quality. However, the accurate estimation of chlorophyll-a con-

Manuscript received September 24, 2009; revised February 21, 2010. Date of publication April 12, 2010; date of current version May 7, 2010. This work was supported in part by the Ministry of Education, Culture, Sports, Science and Technology from Japan under Grants-in-Aid for Scientific Research 20510003 and 19404012 and in part by the National Natural Science Foundation of China under Grant 40871162.

W. Yang is with the Graduate School of Life and Environmental Sciences, University of Tsukuba, Tsukuba 305-8572, Japan, and also with the State Key Laboratory of Earth Surface Processes and Resource Ecology, Beijing Normal University, Beijing 100875, China (e-mail: yangwei1022@gmail.com).

B. Matsushita and T. Fukushima are with the Graduate School of Life and Environmental Sciences, University of Tsukuba, Tsukuba 305-8572, Japan (e-mail: mbunkei@sakura.cc.tsukuba.ac.jp; fukusima@sakura.cc.tsukuba.ac.jp)

J. Chen is with the State Key Laboratory of Earth Surface Processes and Resource Ecology, Beijing Normal University, Beijing 100875, China (e-mail: chenjin@ires.cn).

R. Ma is with the State Key Laboratory of Lake Science and Environments, Nanjing Institute of Geography and Limnology, Chinese Academy of Sciences, Nanjing 210008, China (e-mail: rhma@niglas.ac.cn).

Digital Object Identifier 10.1109/LGRS.2010.2044364 centration (Chl-a) from remotely sensed data is particularly challenging in case-II waters (waters containing not only phytoplankton but also suspended sediments, dissolved organic matter, and anthropogenic substances whose concentrations are not correlated with Chl-a). Therefore, substantial efforts have been made to improve the estimation accuracy in case-II waters [1].

Recently, a conceptual three-band model has been proposed for determining Chl-a in case-II waters [2]. This model requires reflectance in three spectral bands $\left(\lambda_{1}=660-670 \mathrm{~nm}, \lambda_{2}=\right.$ $700-730 \mathrm{~nm}$, and $\lambda_{3}=740-760 \mathrm{~nm}$ ) as input to calculate a three-band index. The selection of these bands is based on the following three requirements [2]: 1) The first band $\lambda_{1}$ should be maximally sensitive to phytoplankton absorption; 2) the second band $\lambda_{2}$ should be minimally sensitive to phytoplankton absorption, but tripton and colored dissolved organic matter (CDOM) absorption should be similar to those in the first band; and 3) the third band $\lambda_{3}$ should be minimally affected by the absorption of phytoplankton, tripton, and CDOM but should have similar backscattering to those of the first and second bands. Given these requirements, the three-band index can be expressed as follows:

$$
\begin{aligned}
{\left[1 / R_{\mathrm{rs}}\left(\lambda_{1}\right)-1 / R_{\mathrm{rs}}\left(\lambda_{2}\right)\right] \times R_{\mathrm{rs}}\left(\lambda_{3}\right) } \\
=\left[a_{\mathrm{ph}}\left(\lambda_{1}\right)+a_{w}\left(\lambda_{1}\right)-a_{w}\left(\lambda_{2}\right)\right] / a_{w}\left(\lambda_{3}\right)
\end{aligned}
$$

where $R_{\mathrm{rs}}$ is the above-water remote-sensing reflectance and $a_{\mathrm{ph}}$ and $a_{w}$ are the absorption coefficients for phytoplankton and pure water, respectively. The three optimal bands are available on the Medium Resolution Imaging Spectrometer (MERIS) sensor, allowing the application of the three-band model to real MERIS data.

However, Le et al. [3] pointed out that one of the assumptions of the original three-band model that the absorption and backscattering of suspended solids can be neglected at $\lambda_{3}$ is not reasonable in some highly turbid case-II waters such as those of Lake Taihu, China. Therefore, the original three-band index for highly turbid case-II waters should be expressed as follows:

$$
\begin{aligned}
& {\left[1 / R_{\mathrm{rs}}\left(\lambda_{1}\right)-1 / R_{\mathrm{rs}}\left(\lambda_{2}\right)\right] \times R_{\mathrm{rs}}\left(\lambda_{3}\right)} \\
& \quad=\left[a_{\mathrm{ph}}\left(\lambda_{1}\right)+a_{w}\left(\lambda_{1}\right)-a_{w}\left(\lambda_{2}\right)\right] /\left[a_{w}\left(\lambda_{3}\right)+b_{b}\left(\lambda_{3}\right)+a_{d}\left(\lambda_{3}\right)\right]
\end{aligned}
$$

where $a_{d}$ is the absorption coefficient of the water constituents excluding pure water and $b_{b}$ is the total backscattering coefficient. To further remove (or minimize) the absorption and backscattering effects of suspended solids and also to partially 
suppress the absorption of pure water at the denominator of (2), Le et al. [3] developed a four-band index instead of the original three-band index suggested in [2]. The four-band index can be expressed as follows:

$$
\begin{aligned}
& {\left[1 / R_{\mathrm{rs}}\left(\lambda_{1}\right)-1 / R_{\mathrm{rs}}\left(\lambda_{2}\right)\right] /\left[1 / R_{\mathrm{rs}}\left(\lambda_{4}\right)-1 / R_{\mathrm{rs}}\left(\lambda_{3}\right)\right]} \\
& \quad=\left[a_{\mathrm{ph}}\left(\lambda_{1}\right)+a_{w}\left(\lambda_{1}\right)-a_{w}\left(\lambda_{2}\right)\right] /\left[a_{w}\left(\lambda_{4}\right)-a_{w}\left(\lambda_{3}\right)\right] .
\end{aligned}
$$

The four-band index was found to provide a better estimation of Chl-a than the original three-band index in the case of Lake Taihu, China [3]. As a crucial prerequisite, successful application of the four-band model depends on the availability of reflectance data at four narrow bands within the wavelength range of $660-760 \mathrm{~nm}$. However, in practice, it is difficult to satisfy this prerequisite from existing satellite sensors.

The objectives of the present study are the following: 1) to enhance the original three-band index by adapting the principle of a four-band index and 2) to evaluate the performance of the enhanced three-band index using the data collected from two turbid Asian lakes, namely, Lake Kasumigaura, Japan, and Lake Dianchi, China.

\section{DATA AND Methods}

\section{A. In Situ and Laboratory Data Collection}

Water samples and corresponding reflectance spectra were collected from two lakes in Japan and China. Lake Kasumigaura is located in the eastern part of Japan's Kanto Plain and has a surface area of $171 \mathrm{~km}^{2}$, an average depth of $4 \mathrm{~m}$, and a maximum depth of $7.3 \mathrm{~m}$; Lake Dianchi is located in a plateau area of southwestern China and has a surface area of $300 \mathrm{~km}^{2}$, an average depth of $4.3 \mathrm{~m}$, and a maximum depth of $11.3 \mathrm{~m}$. Four data collection campaigns were carried out in February 2006 (10 sites), in September 2007 (11 sites), in August 2008 (14 sites), and in September 2009 (21 sites) in Lake Kasumigaura. Another four campaigns were carried out in October 2007 (3 sites), in July 2008 (5 sites), and in March and July 2009 (4 and 41 sites, respectively) in Lake Dianchi. Reflectance measurements were performed between 10:00 and 14:00 h local time. All measurements were taken over optically deep water, and floating scum was not found at these sampling sites. The water-leaving radiance $\left(L_{u}(\lambda)\right)$, the downward irradiance $\left(E_{d}(\lambda)\right)$, and the downward radiance of skylight $\left(L_{\text {sky }}(\lambda)\right)$ were measured at each site using a FieldSpec HandHeld spectroradiometer (Analytical Spectral Devices, Inc., Boulder, CO) in the range of 325-1075 nm at $1-\mathrm{nm}$ intervals. The above-water remote-sensing reflectance $\left(R_{\mathrm{rs}}(\lambda)\right)$ was calculated approximately using the following equation [4]:

$$
R_{\mathrm{rs}} \approx\left(\frac{L_{u}(\lambda)}{E_{d}(\lambda)}-\frac{F(\theta) L_{\mathrm{sky}}(\lambda)}{E_{d}(\lambda)}\right) \times \operatorname{Cal}(\lambda) \times 100
$$

where $\operatorname{Cal}(\lambda)$ is the spectral reflectance calibration factor for the Spectralon reflectance panel (SRT-99-100). $F(\theta)$ represents the reflectance of the skylight at the air-water interface ranging from 0.022 to 0.025 , and $\theta$ is the measurement view angle [5]. In this study, a constant value of 0.024 was used for $F(\theta)$.

Chlorophyll-a was extracted using methanol $(100 \%)$ at $4{ }^{\circ} \mathrm{C}$ for $24 \mathrm{~h}$ under dark conditions. The absorbances of the extracted chlorophyll-a were measured at four wavelengths $(750,663$, 645 , and $630 \mathrm{~nm}$ ), and the concentrations were calculated according to Scientific Committee on Oceanic Research-United Nations Educational, Scientific and Cultural Organization equations [6]. CDOM absorption was measured using a Shimadzu UV-1700 spectrophotometer after water was filtered. The concentrations of the total suspended solids (TSSs), organic suspended solids (OSSs), and inorganic suspended solids (ISSs) were determined gravimetrically. Samples were filtered through precombusted Whatman $\mathrm{GF} / \mathrm{F}$ filters at $500^{\circ} \mathrm{C}$ for $4 \mathrm{~h}$ to remove dissolved organic matter in suspension, which was then dried at $105{ }^{\circ} \mathrm{C}$ for $4 \mathrm{~h}$ and weighed to obtain TSS. The filters were recombusted at $500{ }^{\circ} \mathrm{C}$ for $4 \mathrm{~h}$ and then weighed again to obtain ISS. OSS was derived by subtracting ISS from TSS.

\section{B. Development of an Enhanced Three-Band Index and Accuracy Assessment}

For the original three-band index, the following assumptions were presented by Gitelson et al. [2]:

Assumption 1) $a_{\mathrm{ph}}\left(\lambda_{1}\right) \gg a_{\mathrm{ph}}\left(\lambda_{2}\right), a_{\mathrm{tr}}\left(\lambda_{1}\right) \approx a_{\mathrm{tr}}\left(\lambda_{2}\right)$, and $a_{\text {cdom }}\left(\lambda_{1}\right) \approx a_{\text {cdom }}\left(\lambda_{2}\right)$

Assumption 2) $a_{w}\left(\lambda_{3}\right) \gg a_{\mathrm{ph}}\left(\lambda_{3}\right)+a_{\mathrm{tr}}\left(\lambda_{3}\right)+a_{\mathrm{cdom}}\left(\lambda_{3}\right)$;

Assumption 3) $b_{b}\left(\lambda_{1}\right) \approx b_{b}\left(\lambda_{2}\right) \approx b_{b}\left(\lambda_{3}\right)$.

Here, $a_{\mathrm{tr}}$ and $a_{\text {cdom }}$ are the absorption coefficients for tripton and CDOM, respectively. Accordingly, the original three-band index written as (1) can remove the effects of tripton and CDOM. However, in highly turbid case-II waters, assumption 2 is violated, and the original three-band index should be rewritten as (2). The remaining effects of tripton and CDOM in (2) resulted in uncertainty in estimating Chl-a [3]. On the other hand, comparing the characteristics of the absorption coefficients of phytoplankton, tripton, and CDOM between the second and third bands suggested by Gitelson et al. [2], it is found that their absorptions in the second band are similar to those in the third band (i.e., $a_{\mathrm{ph}}\left(\lambda_{2}\right) \approx a_{\mathrm{ph}}\left(\lambda_{3}\right), a_{\mathrm{tr}}\left(\lambda_{2}\right) \approx a_{\mathrm{tr}}\left(\lambda_{3}\right)$, and $\left.a_{\text {cdom }}\left(\lambda_{2}\right) \approx a_{\text {cdom }}\left(\lambda_{3}\right)\right)$, even for the highly turbid case-II waters. Therefore, if we adapt the format of the four-band index by using the second band twice, the original three-band index can be modified as

$$
\begin{aligned}
& {\left[1 / R_{\mathrm{rs}}\left(\lambda_{1}\right)-1 / R_{\mathrm{rs}}\left(\lambda_{2}\right)\right] /\left[1 / R_{\mathrm{rs}}\left(\lambda_{3}\right)-1 / R_{\mathrm{rs}}\left(\lambda_{2}\right)\right]} \\
& \quad=\left[a_{\mathrm{ph}}\left(\lambda_{1}\right)+a_{w}\left(\lambda_{1}\right)-a_{w}\left(\lambda_{2}\right)\right] /\left[a_{w}\left(\lambda_{3}\right)-a_{w}\left(\lambda_{2}\right)\right] .
\end{aligned}
$$

It is clear that this modified three-band index (which we designated the enhanced three-band index) has the same physical meaning as the four-band index while holding bands that are identical to those used in the original three-band index. Consequently, the enhanced three-band index overcomes the limitations of the original three-band index (2), particularly in its applications over highly turbid case-II waters.

To evaluate the enhanced three-band index, its performance was compared with that of the original three-band index. For Lake Kasumigaura, the data collected in February 2006, September 2007, and August 2008 (35 sites) were used to establish and calibrate the relationships between Chl-a and the enhanced/original three-band index, while the data collected in September 2009 (21 sites) were used to test the validity of the algorithms. For Lake Dianchi, the data collected in July 2009 (41 sites) were used for algorithm calibration, and the 


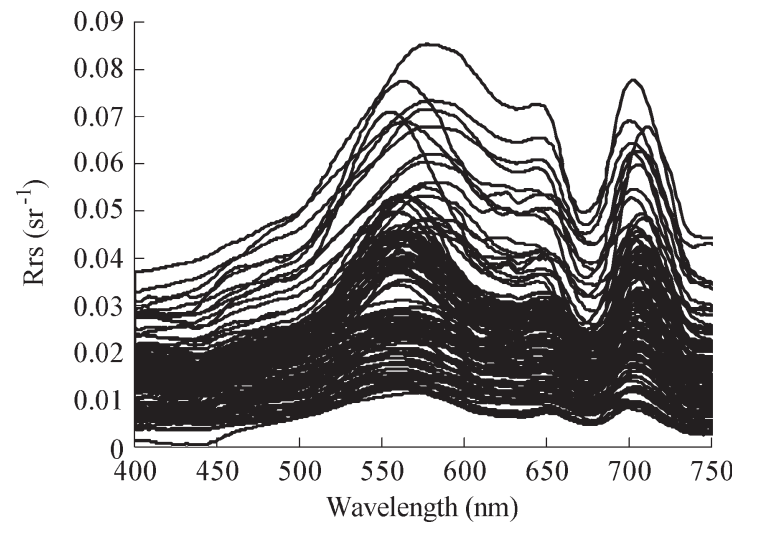

Fig. 1. Remote-sensing reflectance spectra collected in this study.

data collected in October 2007, July 2008, and March 2009 (12 sites in total) were used for algorithm validation. The fieldcollected reflectance spectra were resampled to MERIS bands 7 $(660-670 \mathrm{~nm}), 9(703.75-713.75 \mathrm{~nm})$, and $10(750-757.5 \mathrm{~nm})$ through MERIS spectral response functions to calculate the enhanced and original three-band indexes.

The root-mean-square error (rmse), the mean normalized bias (MNB), and the normalized root-mean-square error (NRMS) were employed to assess the performances of the original and enhanced three-band indexes, which were defined as

$$
\begin{aligned}
r m s e & =\sqrt{\frac{\sum_{i=1}^{N}\left(C h l a_{\text {pred }, i}-C h l a_{\text {meas }, i}\right)^{2}}{N}} \\
M N B & =\operatorname{mean}\left(\varepsilon_{i}\right) \% \\
N R M S & =\operatorname{stdev}\left(\varepsilon_{i}\right) \% \\
\varepsilon_{i} & =100 \times\left(C h l a_{\text {pred }, i}-C h l a_{\text {meas }, i}\right) / C h l a_{\text {meas }, i}
\end{aligned}
$$

where $N$ is the number of samples. $C h l a_{\mathrm{pred}, i}$ and $C h l a_{\text {meas }, i}$ are the predicted and measured Chl-a values for the sample $i$, respectively.

\section{RESULTS}

\section{A. Spectral Properties and Constituent Concentrations}

The magnitude and shape of the reflectance spectra collected in the two lakes, shown in Fig. 1, were quite similar to those for typical case-II waters [2], [3], [7]. Remote-sensing reflectance showed high variability over the visible and near-infrared (NIR) spectral regions. Reflectance in the blue range $(400-500 \mathrm{~nm})$ was very low due to the high absorption of water constituents but was much higher in the green range $(500-600 \mathrm{~nm})$. There was a reflectance trough at around $620 \mathrm{~nm}$ in the spectra because of cyanobacteria that are dominant in the water [8]; a second minimum around $675 \mathrm{~nm}$ corresponded to the red chlorophyll-a absorption maximum. In addition to chlorophylla, the reflectance near $675 \mathrm{~nm}$ was also affected by absorption and scattering by other constituents. A distinct peak located between 690 and $715 \mathrm{~nm}$ appeared in almost all spectra. This peak was from the result of both high backscattering and a minimum in the absorption of all OASs, including pure water [9], [10]. The magnitude of the peak varied widely and was comparable to the magnitude of the green peak for moderate-tohigh Chl-a. Finally, reflectance in the NIR range $(700-750 \mathrm{~nm})$ varied widely and was consistently higher than that in the blue range. In the NIR region of the spectrum, reflectance is mostly controlled by the scattering of all particulate matters.

The data collected in Lake Kasumigaura and Lake Dianchi encompassed varying constituent concentrations. The TSS concentration in Lake Dianchi was very high, averaging 37.11 $\mathrm{g} \cdot \mathrm{m}^{-3}$, and hence, its water was extremely turbid. The TSS concentration of Lake Kasumigaura was somewhat lower, averaging $24.77 \mathrm{~g} \cdot \mathrm{m}^{-3}$. The overall Chl-a of the two lakes was in the range of $22.98-318.6 \mathrm{mg} \cdot \mathrm{m}^{-3}$. The low correlation between TSS and Chl-a clearly indicated that chlorophyll-a was not the only contributor to the optical properties of the waters $\left(R^{2}=0.062\right.$ and $p<0.001$ for Lake Kasumigaura and $R^{2}=$ 0.096 and $p<0.001$ for Lake Dianchi). A low correlation between Chl-a and CDOM was also found in Lakes Kasumigaura $\left(R^{2}=0.053\right.$ and $\left.p<0.001\right)$ and Dianchi $\left(R^{2}=0.079\right.$ and $p<0.001)$. Thus, these two lakes can be classified as case-II waters.

\section{B. Comparison Between the Enhanced and Original Three-Band Indexes}

Fig. 2(a) and (b) shows the calibration results for estimating Chl-a in Lake Kasumigaura. There is a clear and significant linear relationship between the two indexes and Chl-a, while the enhanced three-band index yielded a higher determination coefficient and a smaller rmse $\left(R^{2}=0.90\right.$ with an rmse of $\left.5.59 \mathrm{mg} \cdot \mathrm{m}^{-3}\right)$ than the original one $\left(R^{2}=0.84\right.$ with an rmse of $9.26 \mathrm{mg} \cdot \mathrm{m}^{-3}$ ). For Lake Dianchi, the determination coefficient and rmse of the Chl-a estimation model were also increased and reduced, from 0.84 to 0.91 and from 11.99 to $9.14 \mathrm{mg} \cdot \mathrm{m}^{-3}$, respectively, by using the enhanced three-band index instead of the original one [Fig. 3(a) and (b)]. The slope and intercept of both three-band algorithms were different between Lakes Kasumigaura and Dianchi and also significantly different from the previous study performed in five American lakes and reservoirs (117.4 and 23.1 for slope and intercept, respectively) [11].

For the validation data set, the use of an enhanced threeband index increased the determination coefficient from 0.48 to 0.83 in Lake Kasumigaura. The rmse and random error (NRMS) were also reduced, from 13.97 to $8.68 \mathrm{mg} \cdot \mathrm{m}^{-3}$ and from $19.01 \%$ to $12.30 \%$ [Fig. 2(c) and (d)], respectively. In the case of Lake Dianchi, the rmse and NRMS of the Chl-a estimation were substantially reduced, from 41.29 to $15.82 \mathrm{mg} \cdot \mathrm{m}^{-3}$ and from $35.83 \%$ to $21.43 \%$, respectively, by the use of the enhanced three-band index. The determination coefficient was also increased, from 0.74 to 0.96 [Fig. 3(c) and (d)]. The systematic errors (MNB) were slightly increased for both lakes due to the offset of relative errors $\left[\varepsilon_{i}\right.$ in (9)]. Additionally, Gons et al. [12] proposed a Chl-a estimation algorithm using the reflectance ratio of two MERIS bands (7 and 9) and absorption and backscattering coefficients at these wavelengths. This algorithm was also investigated in the present study using the same data sets for purposes of comparison. The results showed lower accuracy in both lakes compared with the three-band algorithms (the rmse, MNB, and NRMS were $24.97 \mathrm{mg} \cdot \mathrm{m}^{-3},-25.47 \%$, and $12.06 \%$ for Lake Kasumigaura and $50.19 \mathrm{mg} \cdot \mathrm{m}^{-3},-20.06 \%$, and $28.36 \%$ for Lake Dianchi, respectively). 
This article has been accepted for inclusion in a future issue of this journal. Content is final as presented, with the exception of pagination.

(a)

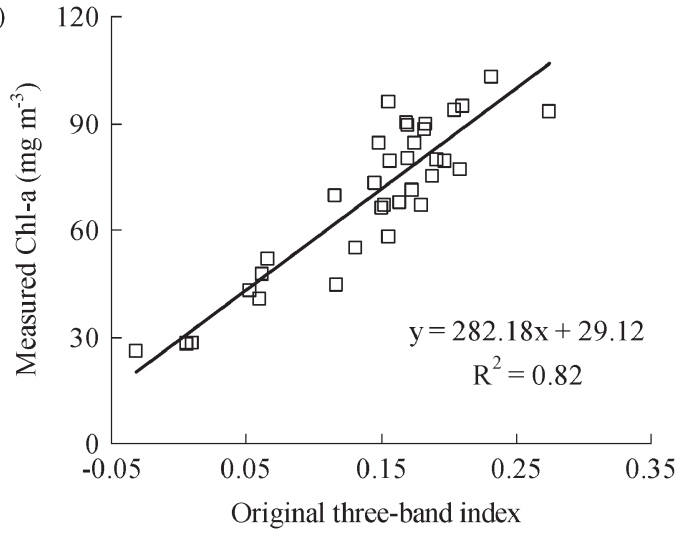

(b)

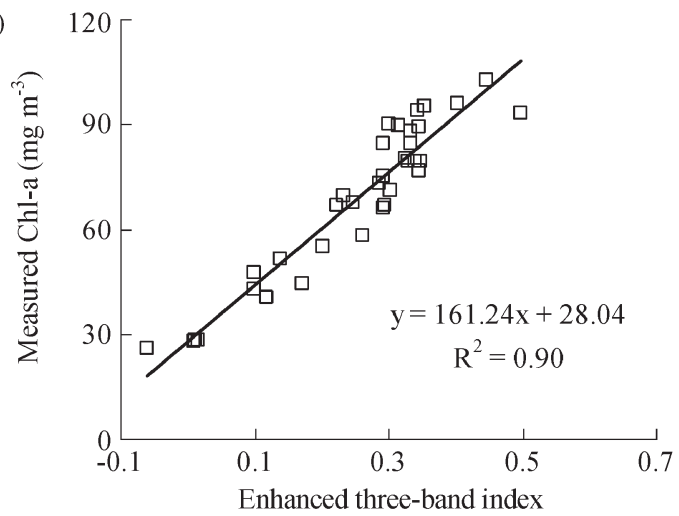

(c)

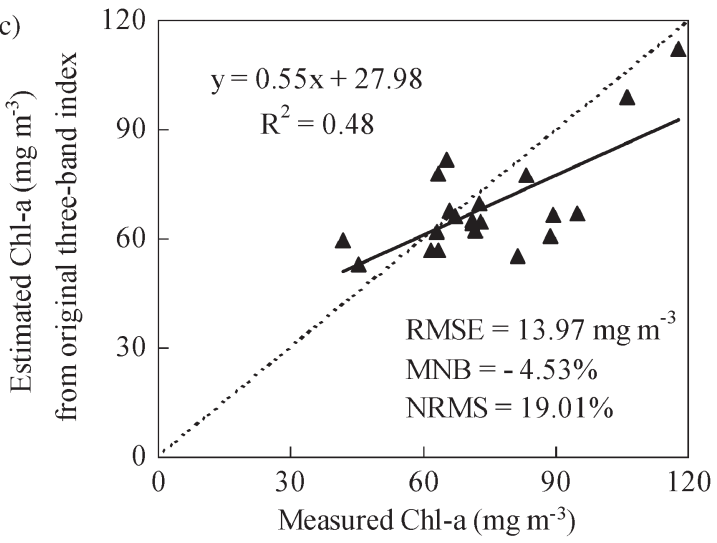

(d)

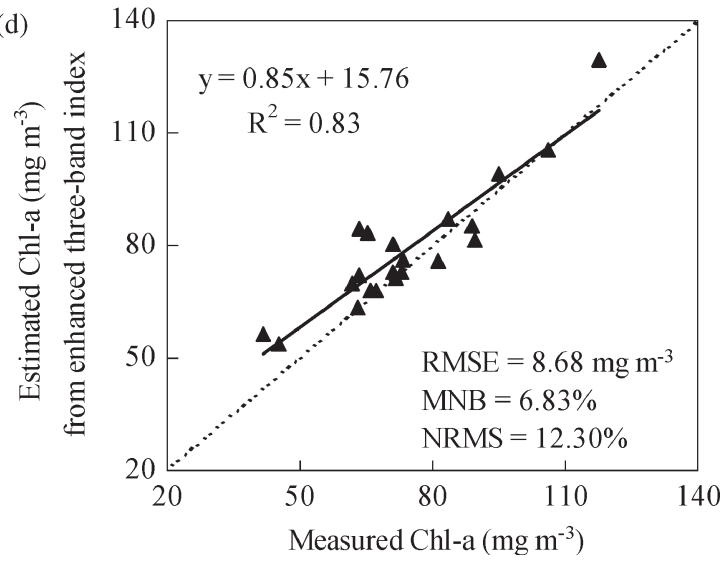

Fig. 2. Calibration and validation of the original/enhanced three-band algorithm for Lake Kasumigaura, Japan. (a) Calibration of the original three-band algorithm. (b) Calibration of the enhanced three-band algorithm (c) Comparison of the measured and estimated Chl-a using the original threeband algorithm. (d) Comparison of the measured and estimated Chl-a using the enhanced three-band algorithm.

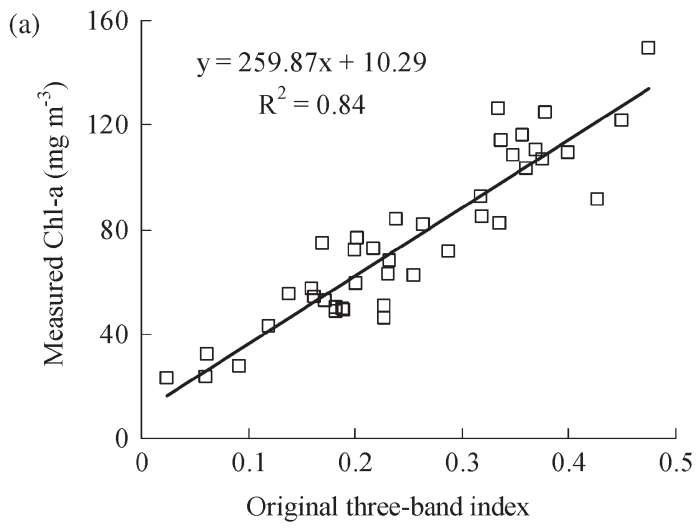

(b)

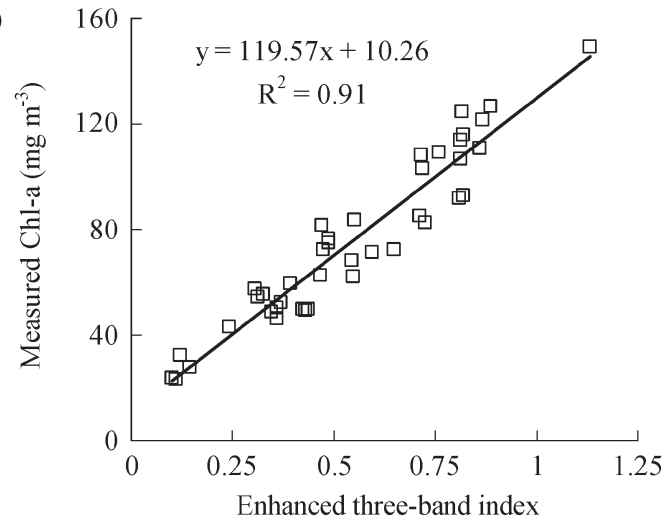

(c)

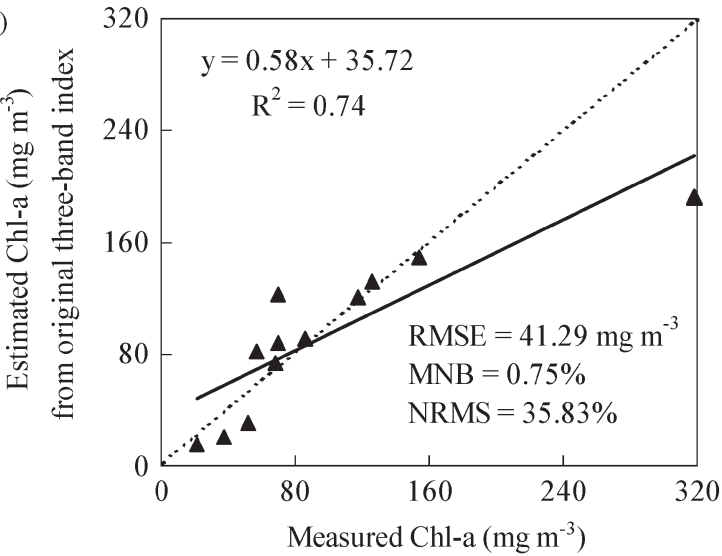

(d)

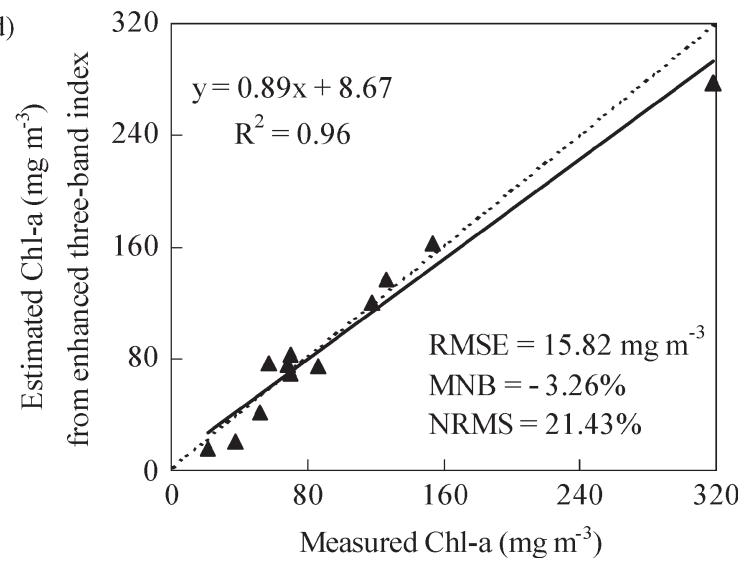

Fig. 3. Calibration and validation of the original/enhanced three-band algorithm for Lake Dianchi, China. (a) Calibration of the original three-band algorithm. (b) Calibration of the enhanced three-band algorithm. (c) Comparison of the measured and estimated Chl-a using the original three-band algorithm. (d) Comparison of the measured and estimated Chl-a using the enhanced threeband algorithm. 


\section{DisCUSSION AND CONCLUSION}

A new three-band index (i.e., the enhanced three-band index) has been proposed to estimate Chl-a in case-II waters. Like the original three-band index, it requires only three bands within the wavelength range of $660-760 \mathrm{~nm}$, which can be provided by the MERIS sensor (bands 7, 9, and 10). Its performance was also improved compared with the original index, particularly in the case of highly turbid case-II waters (e.g., Lake Kasumigaura and Lake Dianchi). One of the assumptions for the original three-band index, that the absorption and backscattering of suspended solids can be neglected at the third band (750$757.5 \mathrm{~nm}$ ), was violated in Lake Kasumigaura and Lake Dianchi. However, the enhanced three-band index successfully overcomes this problem by adapting the principle of the fourband index.

Although the four-band index can also be successfully applied to highly turbid case-II waters, the fact that it requires four narrow bands within the wavelength range of 660-760 nm limits its practical use to the existing satellite sensors. Hyperion is the only satellite hyperspectral sensor that can meet this criterion, but this is still an experimental sensor with a small swath $(7.6 \times 185 \mathrm{~km})$ and a long revisit time and thus cannot be used in routine monitoring. Airborne hyperspectral sensors, such as the Airborne Imaging Spectroradiometer for Applications or the Compact Airborne Visible Spectrographic Imager, can also meet the prerequisite of the four-band index, but the high cost of the data per unit of study area limits their extensive use. In contrast, the MERIS provides the widest capabilities in water monitoring from space, with a spatial resolution of $300 \mathrm{~m}$ and a data acquisition period of three days.

The chlorophyll-a fluorescence emission peak at $685 \mathrm{~nm}$ may impact MERIS bands 7 and 9 used in the three-band index. Nevertheless, Dall'Olmo and Gitelson [13] have shown that the fluorescence has a negligible effect on the formation of the red peak in the reflectance spectra of productive waters. The results of the present study also showed that the enhanced three-band index could estimate Chl-a satisfactorily.

It should be noted that both the slope and intercept of the enhanced three-band algorithm (based on the enhanced threeband index) were clearly different between Lake Kasumigaura and Lake Dianchi [Figs. 2(b) and 3(b)]. This was probably attributable to the different specific inherent optical properties (SIOPs) between these two lakes [14]. These results demonstrated that the mechanism of the enhanced three-band index can remove or minimize the adverse effects of tripton and CDOM for estimating Chl-a, but the index still suffered from effects resulting from phytoplankton itself, such as the variation of SIOPs for chlorophyll-a between the lakes. Challenges still remain in calibrating the algorithm for universal application to different study areas.

The biggest challenge in the application of the enhanced three-band index to real MERIS data is how to remove the atmospheric effects included in the satellite data. The atmosphere usually contributes more than $90 \%$ of the signals measured at the top of the atmosphere. Accurate and reliable atmospheric correction for coastal and inland water imagery is still an unsolvable problem [15]. Dall'Olmo et al. [16] showed that models using red and NIR bands are reasonably resistant to systematic errors due to the atmospheric correction. Nevertheless, Moses et al. [11] demonstrated that the original three-band index was more sensitive to uncertainties in the atmospheric correction procedure than a two-band index, particularly for waters with low reflectance in the NIR region. The enhanced three-band index utilizes the same bands as the original one, and thus, it may still be sensitive to the error due to the atmospheric correction. Further studies will be needed to explore the performance of the enhanced three-band index for retrieving Chl-as from accurately atmospheric-corrected MERIS imagery.

\section{ACKNOWLEDGMENT}

The authors would like to thank four anonymous reviewers for the useful comments on the manuscript.

\section{REFERENCES}

[1] B. Matsushita and T. Fukushima, "Methods for retrieving hydrologically significant surface parameters from remote sensing: A review for applications to East Asia region," Hydrol. Processes, vol. 23, no. 4, pp. 524-533, Feb. 2009.

[2] A. A. Gitelson, G. Dall'Olmo, W. G. Moses, D. C. Rundquist, T. Barrow, T. R. Fisher, D. Gurlin, and J. Holz, "A simple semi-analytical model for remote estimation of chlorophyll-a in turbid waters: Validation," Remote Sens. Environ., vol. 112, no. 9, pp. 3582-3593, Sep. 2008.

[3] C. Le, Y. Li, Y. Zha, D. Sun, C. Huang, and H. Lu, "A four-band semianalytical model for estimating chlorophyll a in highly turbid lakes: The case of Taihu Lake, China," Remote Sens. Environ., vol. 113, no. 6, pp. 1175-1182, Jun. 2009.

[4] Z. Lee, K. L. Carder, C. D. Mobley, R. G. Steward, and J. S. Patch, "Hyperspectral remote sensing for shallow waters. 2. Deriving bottom depths and water properties by optimization," Appl. Opt., vol. 38, no. 18, pp. 3831-3843, Jun. 1999.

[5] C. D. Mobley, "Estimation of the remote-sensing reflectance from abovesurface measurements," Appl. Opt., vol. 38, no. 36, pp. 7442-7455, Dec. 1999.

[6] Determination of Photosynthetic Pigment in Seawater. Monographs on Oceanographic Methodology, SCOR-UNESCO, Paris, France, 1966.

[7] Y. Zhang, M. Liu, B. Qin, H. J. van der Woerd, J. Li, and Y. Li, "Modeling remote-sensing reflectance and retrieving chlorophyll-a concentration in extremely turbid case-2 waters (Lake Taihu, China)," IEEE Trans. Geosci. Remote Sens., vol. 47, no. 7, pp. 1937-1948, Jul. 2009.

[8] S. G. H. Simis, S. W. M. Peters, and H. J. Gons, "Remote sensing of the cyanobacterial pigment phycocyanin in turbid inland water," Limnol. Oceanogr, vol. 50, no. 1, pp. 237-245, Jan. 2005.

[9] A. Gitelson, G. Keydan, and V. Shishkin, "Inland waters quality assessment from satellite data in visible range of the spectrum," Sov. Remote Sens., vol. 6, pp. 28-36, 1985.

[10] A. A. Gitelson, "The peak near $700 \mathrm{~nm}$ on reflectance spectra of algae and water: Relationships of its magnitude and position with chlorophyll concentration," Int. J. Remote Sens., vol. 13, no. 17, pp. 3367-3373, Nov. 1992.

[11] W. J. Moses, A. A. Gitelson, S. Berdnikov, and V. Povazhnyy, "Satellite estimation of chlorophyll-a concentration using the red and NIR bands of MERIS-The Azov sea case study," IEEE Geosci. Remote Sens. Lett., vol. 6, no. 4, pp. 845-849, Oct. 2009.

[12] H. J. Gons, M. Rijkeboer, and K. G. Ruddick, "A chlorophyll-retrieval algorithm for satellite imagery (Medium Resolution Imaging Spectrometer) of inland and coastal waters," J. Plankton Res., vol. 24, no. 9, pp. 947951, Sep. 2002.

[13] G. Dall'Olmo and A. A. Gitelson, "Effect of bio-optical parameter variability and uncertainties in reflectance measurements on remote estimation of chlorophyll-a concentration in turbid productive waters: Modeling results," Appl. Opt., vol. 45, no. 15, pp. 3577-3592, May 2006.

[14] B. Matsushita, W. Yang, J. Chen, and T. Fukushima, "Possibility of improving three-band model for different phytoplankton species in case II water: Evidences from three experiments," J. Remote Sens. Soc. Jpn., vol. 29, no. 5, pp. 653-664, Nov. 2009.

[15] T. Kutser, "Passive optical remote sensing of cyanobacteria and other intense phytoplankton blooms in coastal and inland waters," Int. J. Remote Sens., vol. 30, no. 17, pp. 4401-4425, 2009.

[16] G. Dall'Olmo, A. Gitelson, D. Rundquist, B. Leavitt, T. Barrow, and J. Holz, "Assessing the potential of SeaWiFS and MODIS for estimating chlorophyll concentration in turbid productive waters using red and nearinfrared bands," Remote Sens. Environ., vol. 96, no. 2, pp. 176-187, May 2005. 